\title{
Juvenile hyaline fibromatosis: impaired collagen metabolism in human skin fibroblasts
}

\author{
F Breier, S Fang-Kircher, K Wolff, W Jurecka
}

\begin{abstract}
Juvenile hyaline fibromatosis (JHF) is inherited as a fatal autosomal recessive disorder characterised by multiple tumorous mucocutaneous proliferations. In this paper a 14 month old girl with JHF is described. For this condition, a malfunction of collagen synthesis is considered as the pathogenetic cause. Recently published data have revealed an absent band for type III collagen (TIIC) chain in western blot studies of clinically unaffected JHF skin. Therefore supernatants of skin fibroblast cell cultures, obtained from normal human skin, were analysed for type I collagen (TIC) and TIIIC metabolites by radioimmunoassays. Besides the typical morphological connective tissue changes in the skin lesions, TIC synthesis and degradation were found increased in JHF fibroblasts compared with control fibroblasts. In contrast, TIIIC overall metabolism was significantly reduced by $36 \%$ compared with controls. (Arch Dis Child 1997;77:436-440)
\end{abstract}

Keywords: juvenile hyaline fibromatosis; skin; collagen type I and type III metabolism

Juvenile hyaline fibromatosis (JHF) is a rare disease characterised by tumorous skin lesions with the onset in early infancy, joint contractures, thickening of the gums, bone lesions, and tumorous involvement of internal organs. ${ }^{1-11}$ The skin lesions may vary in number and size and represent painful fleshy cutaneous papules, nodules, or tumours mainly located at the neck, elbows, knees, shins, and ankles. The condition is believed to be inherited as an autosomal recessive trait. Consanguinity of the parents has been reported in some of the sporadic cases. Recently, Kayashima et al have suggested JHF to be a connective tissue disorder characterised by increased synthesis of glycosaminoglycans (GAG) by fibroblasts and an impaired type VI collagen metabolism. ${ }^{12}$ In contrast, we demonstrated recently an absent type III collagen (TIIIC) chain in western blot studies of normal skin in this patient with JHF. Therefore we wanted to quantify the synthesis and degradation of type I collagen (TIC) and TIIIC of human skin fibroblasts in JHF. ${ }^{13}$

As all of the affected sites predominantly contain TIC and TIIIC a deregulated metabolism of these collagens might be suspected. In the following paper we present findings that indicate an abnormality of these collagens in a JHF patient.

\section{Case report}

The family history on the mother's side is remarkable: her cousin is suffering from trisomy 21, her nephew shows hyperplasia of the gingiva. In some members of the mother's family contractures of the left hand's fourth finger were observed as individual symptoms (see family tree, fig 1). The parents themselves show no signs of any inherited disease. Both sisters of the patient are healthy. JHF was diagnosed by tumorous plaques on the lateral and medial aspects of the elbows, wrists, knees, and ankles (fig 2), gingival hyperplasia, and papillomatous anal proliferation, and by typical dermatohistopathological and ultrastructural findings.

The patient died at the age of 14 months due to the progression of her disease complicated by pneumonia and respiratory insufficiency. A postmortem examination revealed congestion of the lung and bronchopneumonia, showing a dense peribronchial infiltrate, mostly composed of neutrophils and foamy macrophages. The visceral pleura revealed fibrous thickening.

\section{Experimental}

LIGHT AND ELECTRON MICROSCOPY

Biopsy specimens were taken from tumorous infiltrations of the left ankle under local anaesthesia. Two tissue samples were prepared for light and electron microscopy. For routine histopathology specimens were paraffin embedded and stained with haematoxylin and eosin, periodic acid Schiff (PAS) reagent, and elastica stain. For ultrastructural examination specimens were fixed in $2.5 \%$ glutaraldehyde in $0.1 \mathrm{M}$ phosphate puffer, $\mathrm{pH} 7.2$, postfixed in osmium tetroxide, and embedded in Epon 812. Semithin sections were stained with toluidine blue. Ultrathin sections were stained with lead citrate and uranyl acetate and were analysed in a Jeol 1010 transmission electron microscope.

\section{CELL CULTURE CONDITIONS}

Three further skin biopsy specimens were obtained from skin that appeared clinically normal from the trunk of the patient. Three fibroblast cell lines were grown from these specimens in minimal essential medium supplemented with $10 \%$ fetal calf serum, $1 \%$ non-essential amino acids, $1 \%$ kanamycin, and $1 \%$ glutamine (all Gibco). After five subcultures a total number of $1.5 \times 10^{5}$ cells were incubated in a humidified atmosphere at $37^{\circ} \mathrm{C}$ and $5 \%$ carbon dioxide. After five days in culture, the supernatants were analysed in order to determine collagen and extracellular matrix parameters. 

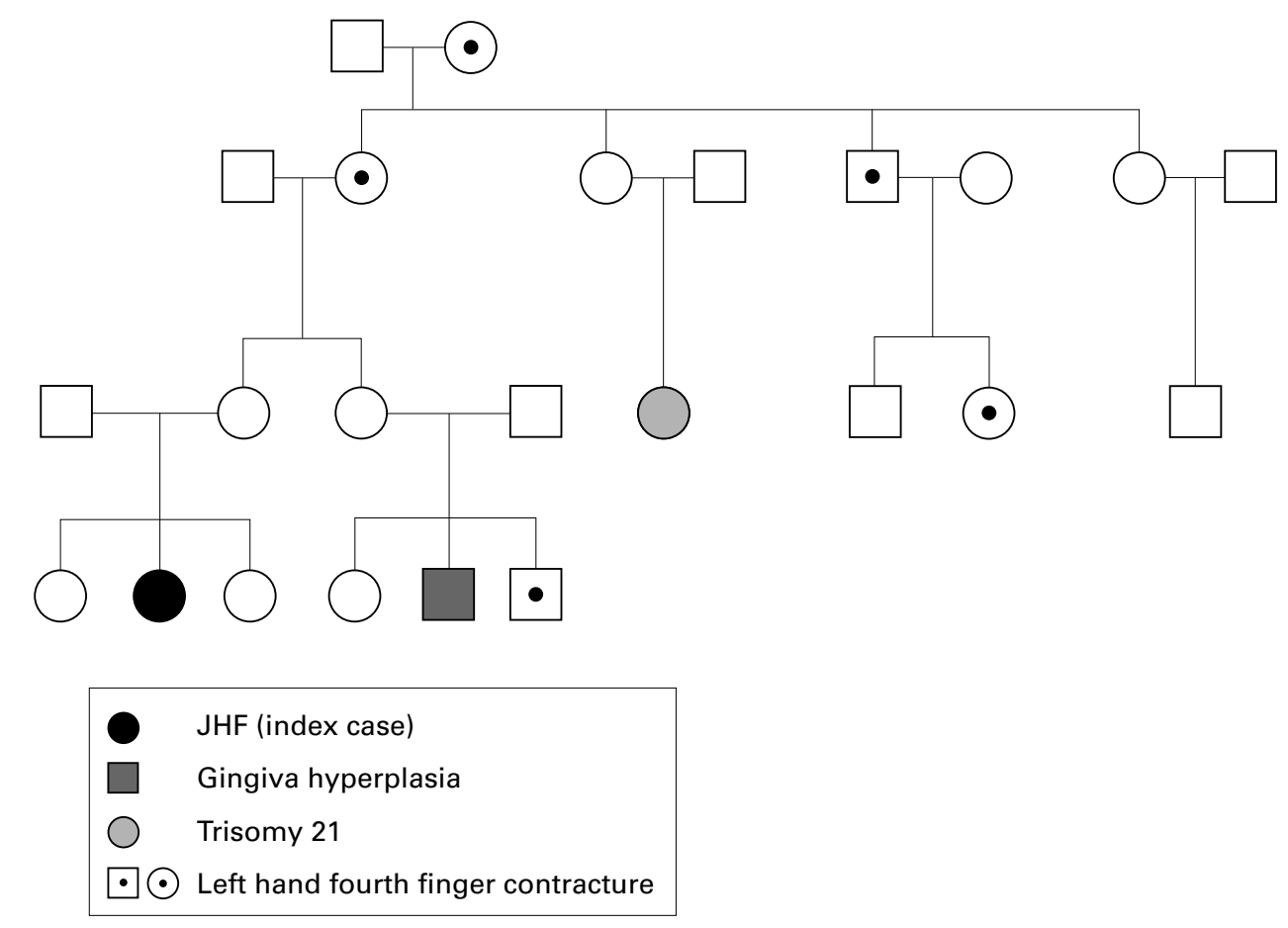

Figure 1 Family tree.

For control purposes three fibroblast cell cultures from clinically normal skin taken from the trunk of three girls (age 12-15 months) undergoing orthopaedic surgery, with no genetically determined disorders, were treated in the same way. Small skin biopsy specimens were taken by informed consent of the patients' parents. Additionally flasks with cell free medium were cultivated. All values of collagen and extracellular matrix parameters in the supernatants of the cell cultures were reduced by the concentrations found in cell free medium to exclude possible influence of fetal calf serum.

ANALYSIS OF EXTRACELLULAR MATRIX PARAMETERS AND TIC AND TIIIC METABOLITES The following parameters were analysed in the supernatants of fibroblast cell cultures: GAG by a spectrophotometric method using dimethylmethylene blue. ${ }^{14}{ }^{15}$ Total protein was measured by a method described by Lowry et al ${ }^{16}$ to exclude methodical interferences in determination of GAG. ${ }^{17}$ Hyaluronic acid was determined by radioimmunoassay (Kabi, Pharmacia, Sweden). In order to investigate collagen metabolites, carboxy terminal propeptide of collagen type I (PICP), carboxy terminal cross linked telopeptide of collagen type I (ICTP), and amino terminal propeptide of collagen type III (PIIINP) were analysed by radioimmunoassay (Orion Diagnostica, USA). PICP and ICTP can be regarded as parameters of synthesis and degradation respectively of collagen type $\mathrm{I}^{18}{ }^{19}$; PIIINP has been reported to reflect the TIIIC overall metabolism. ${ }^{20}{ }^{21}$

Figure 2 Brownish to violaceous fleshy tumorous infiltration of the lateral right ankle with numerous yellowish papules resembling intradermal milia histologically.
All concentrations were correlated with the standardised numbers of cells in each culture flask and were calculated as mean (SD) $\mu \mathrm{g} /$ flask. All parameters of the cell cultures were investigated also in cell free cultivated medium. All the statistical differences were analysed by Student's $t$ test.

\section{Results}

LIGHT MICROSCOPY AND ELECTRON MICROSCOPY OF INVOLVED SKIN (TUMOROUS PLAQUE)

The epidermis showed mild hyperplasia but otherwise regular structures. In the dermis the extracellular component of the connective tissue was markedly increased with irregularly arranged thick collagen bundles and homogeneous eosinophilic matrix (fig 3). There was also increased cellularity, most cells representing fibroblast-like cells with bizarre shape. The skin appendages were reduced in number. The number of small vessels was increased and they were surrounded by concentrically arranged collagen bundles. Similar changes were visible in the mid-dermis and around an epithelial cyst. Elastica stain showed rarefaction of elastic fibres and PAS stain revealed fine granular PAS positive material between the collagen fibres in the homogeneous matrix around the vessels of the dermis. These findings were typical for those already described for tumorous proliferations in JHF.

In semithin sections (fig 4) and electron microscopy (fig 5) the tumorous infiltrations revealed relatively large, bizarre shaped connective tissue cells with pseudopodia-like protrusions. Nuclei were bizarre shaped with numerous invaginations, surrounded by fine granular, electron dense cytoplasm. Most of the cells contained a prominent Golgi apparatus and numerous membrane bound vesicles of varying size up to $1 \mu \mathrm{m}$ in diameter. The vesicles were filled with a fine granular and 


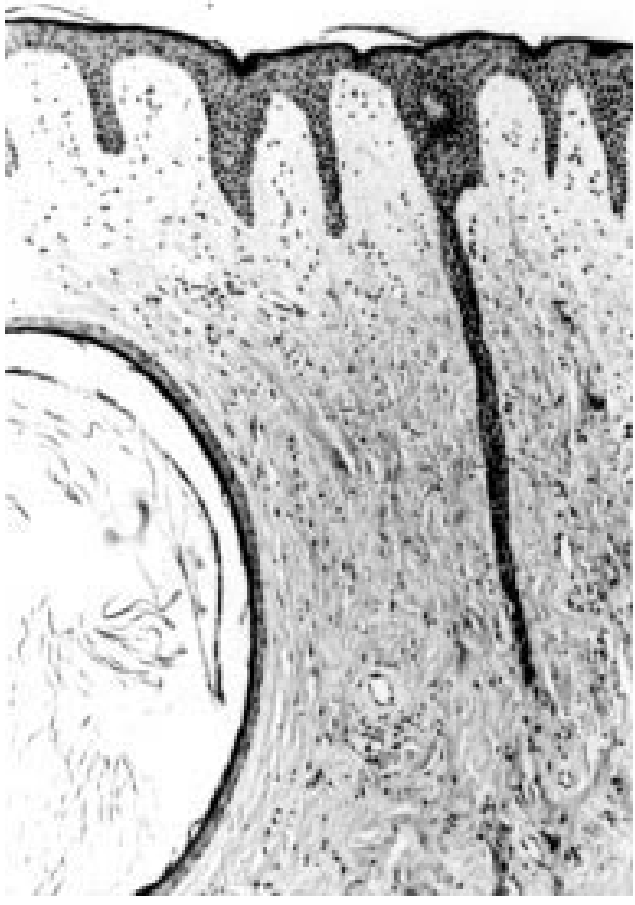

Figure 3 Histology of a biopsy specimen from a tumorous infiltration of the lateral ankle. The dermal connective tissue is increased in thickness by proliferation of connective tisssue cells and extracellular matrix. The vessels and an intradermal epithelial cyst are surrounded by concentrically arranged collagen fibres (paraffin section, haematoxylin and eosin $\times 900$.

microfibrillar material. Similar microfibrillar material was also observed in varying amounts extracellularly between these cells. Additionally some normal mature collagen fibres and long spacing collagen fibres were seen.

CELL CULTURES OF SKIN FIBROBLASTS

(CLINICALLY NORMAL SKIN OF JHF PATIENT AND CONTROLS)

All the cell lines of fibroblasts that were obtained from the patient suffering from JHF showed different growth patterns from those of normal controls. In the confluence stage the control cell lines showed in primary and in subsequent cultures a homogeneous growth pattern with regularly shaped fibroblasts mainly arranged in parallel. However in the confluence stage of the cell cultures of the JHF patient a milky, mucoid pseudomembrane could be observed. Microscopically the cells were irregularly arranged, unevenly orientated, presenting a bizarre growth pattern. Additionally these fibroblasts were uneven in size and shape.

CONCENTRATIONS OF GAG AND HYALURONIC ACID

The supernatants of standardised cell cultures on day 5 showed no significant difference in GAG (69.3 (3.8) $v 73.8$ (9.4) $\mu \mathrm{g} /$ flask) and hyaluronic acid (137.7 (13.6) $v 133.5$ (22.0) $\mu \mathrm{g} /$ flask) in the child with JHF compared with controls (fig 6A).

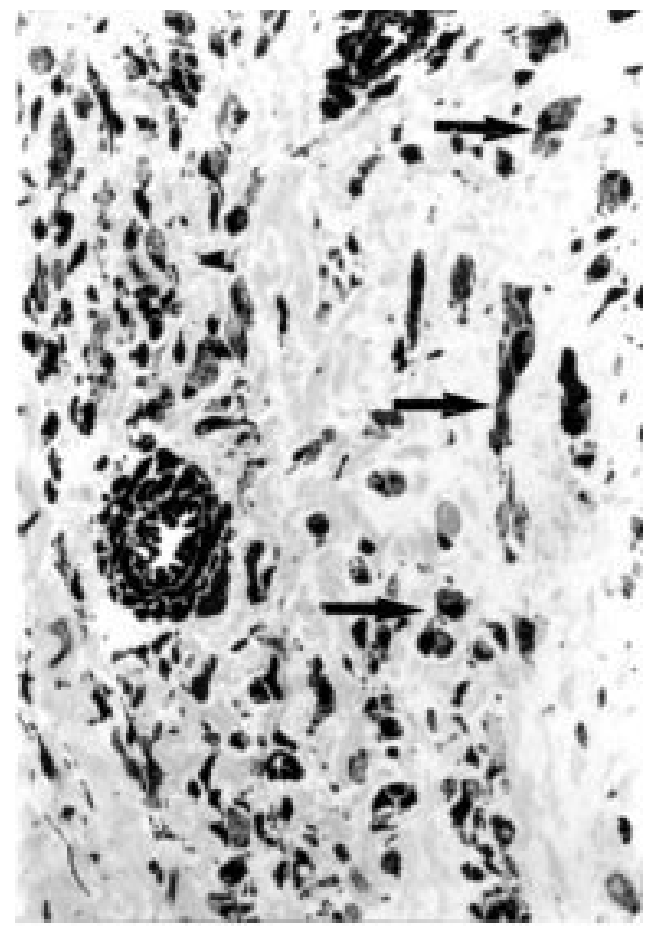

Figure 4 The connective tissue cells are relatively large and bizarre shaped (arrows) and within intracellular vacuoles (semithin section, toluidine blue $\times 3600$ ).

PICP AND ICTP IN FIBROBLAST CELL CULTURES IN JHF

The concentrations of PICP (24.1 (8.4) v 12.6 $(1.0) \mu \mathrm{g} /$ flask, $\mathrm{p}<0.13)$ and ICTP $(0.5(0.1) v$ $0.3(0.1) \mu \mathrm{g} /$ flask; $\mathrm{p}<0.14)$ in the supernatants of the standardised patient's cultures were increased by $91 \%$ and $62 \%$ respectively, compared with the supernatants of standardised cell cultures of the healthy individuals (fig $6 \mathrm{~A})$. However the ratio of both markers (PICP/ ICTP $=45.4$ (7.2) $v 43.1$ (12.3)), reflecting synthesis and degradation of TIC, was equal in both, the pathological and the control samples respectively, indicating an accelerated but yet balanced TIC metabolism (fig 6B).

PIIINP IN FIBROBLAST CELL CULTURES IN JHF PIIINP was highly significant reduced by $36 \%$ in supernatants of standardised JHF fibroblast cultures compared with those of the control cells (1.05 (0.04) $v 1.65$ (0.01) $\mu \mathrm{g} /$ flask; $\mathrm{p}<0.001)$ demonstrating a decreased TIIIC metabolism in JHF (fig 6C). In order to show the differences between the concentrations of TIC and TIIIC metabolites, the ratios of PIIINP/PICP (0.05 (0.02) patient $v 0.13$ (0.01) controls; $\mathrm{p}<0.05)$ and PIIINP/ICTP (2.02 (0.43) patient $v 5.16$ (1.69) controls; $\mathrm{p}<0.05$ ) were calculated (fig $6 \mathrm{C}$ ); both were significant.

\section{Discussion}

In this study we demonstrated a significantly decreased TIIIC and increased TIC synthesis and degradation in fibroblast cell cultures of normal human skin of a patient with JHF. However in JHF several pathogenetic mechanisms have been discussed (table 1). ${ }^{79} 10121323$ Woyke et al speculated on an impaired 


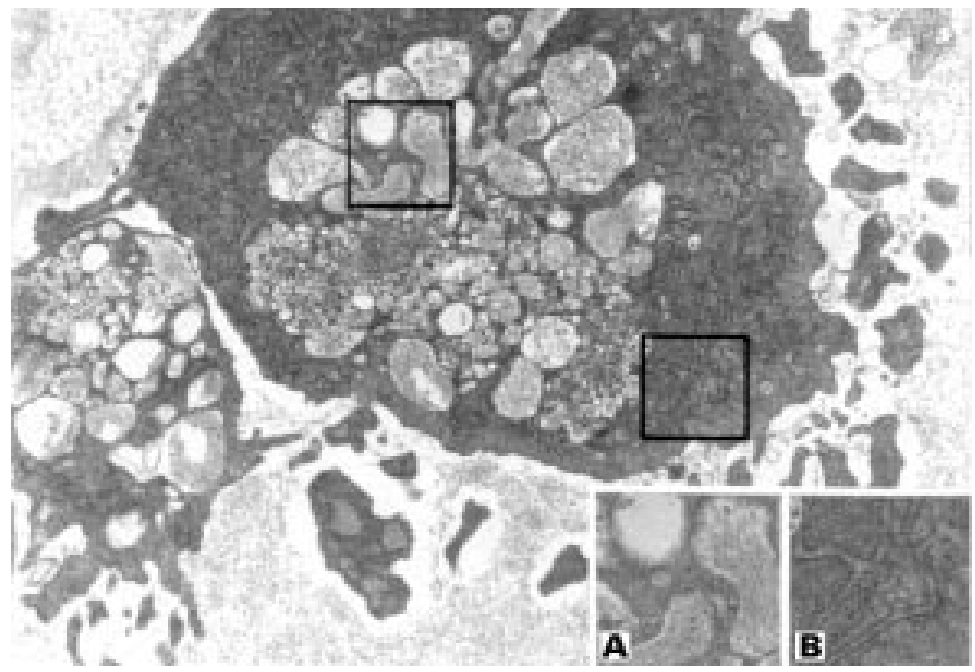

Figure 5 Electron micrograph of a biopsy specimen from a tumorous infiltration showing bizarre shaped fibroblast-like cell containing a prominent Golgi apparatus and numerous membrane bound vesicles with a microfibrillar material in the extracellular matrix (ultrathin section, uranyl acetate, lead citrate $\times 14400$ ). Inset $(A)$ intracytoplasmatic vacuoles containing a microfibrillar and fine granular material and (B) a pronounced Golgi apparatus (magnification $\times 24400)$.

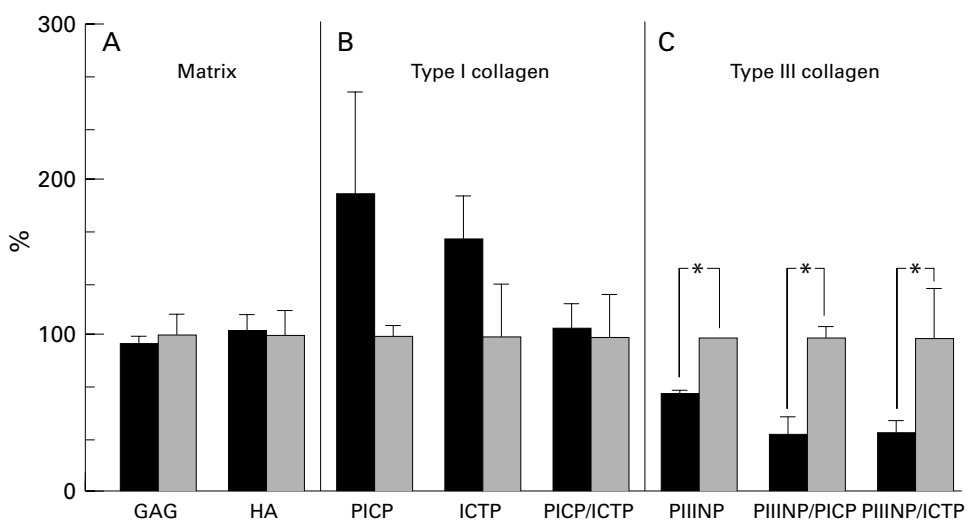

Figure 6 Biochemical analysis. Comparison of extracellular matrix metabolites and TIC and TIIIC metabolism of three biopsy specimens of the patient with $\mathcal{F H F}$ (black bars) and three healthy age matched individuals (shaded bars). Each bar shows the mean (SD) value of the three specimens. All values are shown in percentages whereby the average of the results of healthy (control) skin are regarded as $100 \%$ whereas the values of the $\mathrm{FHF}$ skin fibroblast cultures are expressed as per cent deviation from the controls; asterisks mark significant differences. (A) Of the extracellular matrix glycosaminoglycans (GAG) and hyaluronic acid (HA) were analysed and showed no significant differences. (B) Of TIC metabolism the propeptide of type I collagen (PICP) and carboxy terminal cross linked telopeptide of collagen type I (ICTP) were analysed, showing an increased but yet balanced type I collagen metabolism as indicated by the ratio of PICP/ICTP. (C) Amino terminal propeptide of collagen type III (PIIINP) is a marker for the overall metabolism of TIIIC which is highly significant reduced in $\mathcal{F H F}$ skin compared with normal skin. The ratios PIIINP/PICP and PIIINP/ICTP were calculated to show the significant differences in TIC and TIIIC metabolism in $\mathcal{F H F}$ skin and normal skin respectively.

synthesis of procollagen or tropocollagen, ${ }^{9}$ on the other hand Iwatha et al discussed an increased or faulty synthesis of GAG by fibroblasts leading to this condition..$^{10}$

Further reports suspected increased chondroitin sulphate synthesis or decreased amounts of hyaluronic acid as causative agents. ${ }^{6710}$ However, in contrast to the observations of Iwata et $a l^{10}$ in our patient biochemical investigations of fibroblast cultures' supernatants showed no significant increases of GAG and hyaluronic acid but our investigation was performed on cultures from morphologically unaltered skin.

More recent investigations by Kayashima et al supposed JHF as a disease associated with an
Table 1 Metabolic defects reported in $7 H F$

\begin{tabular}{ll}
\hline Reference & Metabolic defect \\
\hline Woyke $e t a l^{9}$ & Procollagen, tropocollagen \\
Iwatha $e t a l^{10}$ & GAG, hyaluronic acid \\
Kitano $e t a l^{67}$ & Hyaluronic acid, chondroitin sulphate \\
Kayashima $e t a l^{12}$ & Type VI collagen \\
Lubec $e t a l^{13}$ & Type III and type I collagen \\
$\begin{array}{l}\text { Glover } e t a l^{23} \\
\text { Breier } e t a l \\
\quad \text { present study) }\end{array}$ & Type VI collagen \\
\hline
\end{tabular}

impaired collagen type VI metabolism. ${ }^{12}$ However, these findings in our view would not sufficiently explain the clinical spectrum of involvement in JHF, since the skin, the mucous membranes, the joints, and the bones as the major manifestations sites of this condition mainly harbour TIC and TIIIC. Furthermore, recently we demonstrated a deregulation of TIIIC in western blot studies of clinically normal skin in JHF. ${ }^{13}$ The absence of the TIIIC chain accounts for a decreased TIIIC metabolism. TIIIC defects were also described in Ehlers-Danlos syndrome type IV. $^{24}$ A $\operatorname{TIII}\left(\alpha_{1}\right) \mathrm{C}$ defect $^{25-27}$ was reported in vasculopathy in Ehlers-Danlos syndrome leading to a great risk of spontaneous haemorrhage from vascular rupture or bowel perforation. ${ }^{28}$ Recent molecular advances have identified several mutations in the gene for type III procollagen to be responsible for Ehlers-Danlos syndrome type IV. ${ }^{28}$ One gene has been mapped to human chromosome 2q24.3-q31 by in situ hybridisation studies. ${ }^{29}$ We assume that a pathological TIIIC metabolism may be induced by a similar underlying genetic defect in JHF.

Evaluation of TIC metabolites in our patient recorded a markedly increased level of PICP and ICTP but the PICP/ICTP ratio was equal in pathological and control cell lines respectively. This indicates an accelerated, but balanced collagen TIC metabolism. In contrast, concentrations of PIIINP were significantly decreased in supernatants of standardised cultures of JHF cells as consequence of a reduced metabolism and/or release into cell medium. This indicates a decreased TIIIC metabolism in JHF patients and it can be speculated that the reduced enzymatic removal of the TIIIC terminal propeptides, which is necessary for regular cross linking of collagen fibrils, causes a diminished stability of collagen molecules in JHF. Reduced TIIIC overall metabolism may also lead to the intracellular accumulation of mature and immature collagen macromolecules, aggregated in intracellular vacuoles and the extracellular matrix, which was demonstrated by Woyke et al ${ }^{\rho}$ and by us.

Histopathological investigations of clinically involved skin showed increased cellularity of the dermal connective tissue cells extending from the papillary dermis to the subcutaneous fat and deposits of increased amounts of cell fibres. Ultrastructural analysis revealed, according to the findings of Woyke et al, ${ }^{9}$ numerous fibroblast-like cells containing a highly active Golgi apparatus. It is generally believed that vesicles of the Golgi apparatus of a fibroblast contain a solution of monomeric tropocollagen macromolecules which are precursors 
of stroma collagen. These macromolecules have to be secreted from the Golgi apparatus to the extracellular space where soluble tropocollagen units are quickly transformed into fibrils. ${ }^{9}$ Excess of such a floccular and fibrillar material can be detected between the numerous cells of the skin tumours, indicating that the basic defect is a localised metabolic disturbance in the formation of collagen. In adult human dermis TIC accounts for approximately $80 \%$ of total collagen..$^{30}$ The synthesis of TIC accelerates during the neonatal period until the ratio of TIC toTIIIC in adult human skin is $6: 1 .^{30}$ Western blot analysis of our patient showed an absent TIIIC chain exclusively in the skin, but not in other organs, such as heart, liver, and kidney. ${ }^{13}$ This may be explained by a decreased synthesis of TIIIC in skin fibroblasts.

It can be assumed that the increased TIC turnover and the excessive production of the extracellular matrix are secondary effects due to the disturbed TIIIC metabolism which obviously is fundamentally involved in the pathogenesis of this disorder. The suggestion, that the presence of type III procollagen, in particular, at the surface of collagen fibrils has a fibril diameter limiting role for TIC, underlines this hypothesis. ${ }^{31}$ This may be an explanation for the clinical presentation of tumorous infiltrates with predominance above mechanically strained sites.

In conclusion these results reflect a decreased TIIIC metabolism with a possibly secondarily increased TIC metabolism and may be regarded as the reason for clinical manifestations of JHF.

1 Murray J. On three peculiar cases of molluscum fibrosum in children. Medico Chirurgical Transactions London 1873;38:235-53.

2 Roggli VL, Kim HS, Hawkins E. Congenital generalized fibromatosis. Cancer 1980;45:954-60.

3 Finlay AY, Ferguson SD, Holt PJA. Juvenile hyaline Finlay AY, Ferguson SD, Holt PJA. Juvenil

4 Fayad MN, Yacaoub A, Salman S, Khudr A, Der Kaloustian Fayad MN, Yacaoub A, Salman S, Khudr A, Der Kaloustian VM. Juvenile hyaline fibromatosis: two new patients and
review of the literature. Am $\mathcal{F}$ Med Genet 1987;26:123-31.

5 Quintal D, Jackson R. Juvenile hyaline fibromatosis. Arch Quintal D, Jackson R. Juvenile
Dermatol 1985;121:1062-3.

Dermatol 1985;121:1062-3.
Kitano Y, Horiki M, Aoki T, Sagami S. Two cases of juvenile hyaline fibromatosis: some histological, electron microscopic and tissue culture observations. Arch Dermato 1972;106:877-83

7 Kitano Y. Juvenile hyaline fibromatosis. Arch Dermato 1976;112:86-8

8 Ishikawa $\mathrm{H}$, Meada $\mathrm{H}$, Takamatsu $\mathrm{H}$, Saito Y. Systemic hyalinosis (juvenile hyaline fibromatosis): ultrastructure of the hyaline with particular reference to the cross banded structure. Arch Dermatol 1979;265:195-206.

9 Woyke S, Dogmagala W, Olszewski W. Ultrastructure of a fibromatosis hyalinica multiplex juvenilis. Cancer 1970;26: 1157-86.

10 Iwata S, Horiuchi R, Maeda H, Ishikawa H. Systemic hyalinosis or juvenile hyaline fibromatosis: ultrastructure and biochemical study of culture of skin fibroblasts. Arch Dermatol Res 1980;267:115-21.

11 Drescher E, Woyke S, Markiewicz C, Tegi S. Juvenile fibromatosis in siblings. F Pediatr Surg 1967;2:427-30

12 Kayashima K, Katagiri K, Shinkai H, Ono T. Is juvenile hyaline fibromatosis a type collagen VI collagenosis? In: shibashi Y, Nakagawa H, Suzuki H, eds. Electron microscopy in dermatology-basic and clinical research. Amsterdam: Excerpta Medica, 1994: 329-34.

13 Lubec B, Steinert I, Breier F, Jurecka W, Pillwein K, FangKircher S. Skin collagen defects in a patient with juvenile hyaline fibromatosis. Arch Dis Child 1995;73:246-8.

14 de Jong JGN, Wevers RA, Laarakkers C, Poorthuis BJHM. Dimethylmethylene blue-based spectrophotometry of glycosaminoglycans in untreated urine: a rapid screening procedure for mucopolysaccharidoses. Clin Chem 1989;35: $1472-7$

15 Sabiston P, Adams ME, Ho YA. Automation of 1,9dimethylmethylenblue blue dye-binding assay for sulfated glycosaminoglycans with application to cartilage microcultures. Anal Biochem 1985;149:543-8

16 Lowry OH, Rosebrough NJ, Farr AL, Randall RJ. Protein measurment with the folin phenol reagent. 7 Biol Chem 1951;193:265-75.

17 Whitley CB, Ridnour MD, Draper KA, Dutton CM, Negila JP. Diagnostic test for mucopolysaccharidosis I. Direct method for quantifying excessive urinary glycosaminoglymethod for quantifying excessive urinary

18 Prockop DJ, Kivirikko KI, Tuderman L, Guzman NA. The biosynthesis of collagen and its disorders. $N$ Engl f Med 979;301:77-85.

19 Risteli J, Elomaa I, Niemi S, Novamo A, Risteli L. Radioimmunoassay for the pyridinoline cross-linked carboxyterminal telopeptide of type I collagen: a new serum marker of bone resorption. Clin Chem 1993;39:635-40.

20 Risteli L, Risteli J. Radioimmunoassays for monitoring connective tissue metabolism. Rheumatology 1986;10:216-45.

21 Risteli L, Risteli J. Analysis of extracellular matrix proteins in biological fluids. Methods Enzymol 1987;145:391-411.

22 McKusick VA. Fibromatosis, juvenile hyaline [Puretic syndrome, included; hyalinosis, systemic juvenile, included] Mendelian inheritance in man. 10th Ed. Vol 2. Baltimore and London: John Hopkins University Press, 1992: more

23 Glover MT, Lake BD, Atherton DJ. Infantile systemic hyalinosis:newly recognized disorder of collagen? Pediatrics 1991;87:228-34

24 Mackay K, Raghunath M, Superti-Furga A, Steinmann B, Dalgleish R. Ehlers-Danlos syndrome type IV caused by Gly400Glu, Gly595Cys and Gly1003Asp substitutions in collagen III: clinical features, biochemical screening, and molecular confirmation. Clin Genet 1996;49:286-95.

25 Pope FM, Narcisi P, Nicholls AC, Germaine D, Pals G Richards AJ. COL3A1 mutations cause variable clinical phenotypes including acrogeria and vascular rupture. $\mathrm{Br} \mathcal{F}$ phenotypes including acrogeri

26 Germain D. Ehlers-Danlos syndromes. Clinical, genetic and molecular aspects. Ann Dermatol Venereol 1995;122:187204.

27 Chiodo AA, Sillence DO, Cole WG, Bateman JF. Abnormal type III collagen produced by an exon-17-skipping mutation of the COL3A1 gene in Ehlers-Danlos syndrome type IV is not incorporated into the extracellular matrix. Biochem f 1995;311:939-43.

28 Kuivaniemi H, Tromp G, Bergfeld WF, Kay M, Helm TN. Ehlers-Danlos syndrome type IV: a single base substitution of the last nucleotide of exon 34 in COI $3 \mathrm{~A} 1$ leads to exon skipping. F Invest Dermatol 1995;105:352-6.

29 Tiller GE, Polumbo PA, Summar ML. Linkage mapping of the gene for type III collagen (COL3A1) to human chromosome 2q using a VNTR polymorphism. Genomics 1994;20:275-7.

30 Uitto J. Biology of dermal cells and extracellular matrix. Scleroderma. In: Fitzpatrick TB, Eisen AZ, Wolff K, Freedberg IM, Austen FK, eds. Dermatology in general medicine. 4th Ed. New York: McGraw-Hill, 1993: 299-315.

31 Kielty CM, Hopkinson I, Grant ME. Collagen: the collagen family: structure, assembly, and organization in the extracellular matrix. In: Royce PM, Steinmann B, eds. Connective tissue and inheritable disorders. Molecular, genetic, and medical aspects. New York: 1992: 103-47. 